\title{
Antiangiogenic Role of Somatostatin Receptor 2 in a Model of Hypoxia-Induced Neovascularization in the Retina: Results from Transgenic Mice
}

\author{
Massimo Dal Monte, ${ }^{1}$ Maurizio Cammalleri, ${ }^{1}$ Davide Martini, ${ }^{1,2}$ Giovanni Casini, ${ }^{2}$ and \\ Paola Bagnoli
}

Purpose. To determine whether the somatostatin receptor 2 $\left(\mathrm{sst}_{2}\right)$ influences angiogenesis and its associated factors in a model of hypoxia-induced retinal neovascularization.

Methods. sst $_{1}$-knockout (KO) mice, in which $\mathrm{sst}_{2}$ is overexpressed and overfunctional, and $\mathrm{sst}_{2}-\mathrm{KO}$ mice were used. Angiogenesis was evaluated in fluorescein-perfused retinas. Angiogenesis-associated factors were determined by RT-PCR and immunohistochemistry.

Results. Retinal neovascularization was increased in $\mathbf{s s t}_{2}-\mathrm{KO}$ mice, but remained unchanged in $\mathrm{sst}_{1}-\mathrm{KO}$ compared with wild-type (WT) mice. Retinal levels of $\mathrm{sst}_{2}$ mRNA were not affected by hypoxia. Normoxic levels of angiogenesis regulators were similar in WT and KO retinas except for mRNA levels of IGF-1, Ang-2, and its receptor Tie-2. In WT, hypoxia induced an increase in mRNA levels of (1) VEGF and its receptors, (2) IGF-1R, and (3) Ang-2 and Tie-2. The increase in VEGF and IGF-1R mRNAs was more pronounced after sst $_{2}$ loss, but was less pronounced when $s t_{2}$ was overexpressed. In addition, in hypoxic retinas, $\mathrm{sst}_{2}$ loss increased IGF-1 mRNA, whereas it decreased Ang-1, Tie-1, and Tie-2 mRNA levels. Moreover, Tie-1 mRNA increased when $s t_{2}$ was overexpressed. Immunohistochemistry confirmed the results in hypoxic retinas on increased expression of VEGF, IGF-1, and their receptors after $\mathrm{sst}_{2}$ loss. It also allowed the localization of these factors to specific retinal cells. In this respect, VEGFR-2, IGF-1, and IGF-1R were localized to Müller cells.

Conclusions. These results suggest that $\mathrm{sst}_{2}$ may be protective against angiogenesis. The immediate clinical importance lies in the establishment of a potential pharmacological target based on sst $_{2}$ pharmacology. (Invest Ophthalmol Vis Sci. 2007;48: 3480 -3489) DOI:10.1167/iovs.06-1469

$\mathrm{T}$ he abnormal formation of new blood vessels characterizes a variety of retinal diseases, including diabetic retinopathy, ${ }^{1}$ and requires the involvement of vascular endothelial growth factor (VEGF), insulin-like growth factor (IGF)-1, and their receptors VEGFR-1, VEGFR-2, and IGF-1R. Although the

From the ${ }^{1}$ Dipartimento di Biologia, Università di Pisa, Via san Zeno, Pisa, Italy; ${ }^{2}$ Dipartimento di Scienze Ambientali, Università della Tuscia, Largo dell'Università, Viterbo, Italy.

Supported by Grant 2005052312 from the Italian Ministry of University and Research (MUR, PRIN) and Grant 2006-0146 Fondazione Cassa di Risparmio di Volterra.

Submitted for publication December 12, 2006; revised March 8, 2007; accepted May 21, 2007.

Disclosure: M. Dal Monte, None; M. Cammalleri, None; D. Martini, None; G. Casini, None; P. Bagnoli, None

The publication costs of this article were defrayed in part by page charge payment. This article must therefore be marked "advertisement" in accordance with 18 U.S.C. $\$ 1734$ solely to indicate this fact.

Corresponding author: Paola Bagnoli, Dipartimento di Biologia, Università di Pisa, Via san Zeno, 31, 56127 Pisa, Italy;

pbagnoli@biologia.unipi.it. available information on their expression in the retina is far from being exhaustive, these factors have been localized to both retinal cells and microvascular endothelium. ${ }^{2-6}$ Retinal neoangiogenesis is always associated with an increase of VEGF and its receptors ${ }^{2,3,7}$ but not of IGF-1. ${ }^{8-10}$ Of the other downstream factors affecting blood vessel growth, recent results indicate that Ang-1 and -2 and their tyrosine kinase receptor Tie- 2 are regulated by hypoxia and play a role in retinal neovascularization. ${ }^{11,12}$ Although a ligand for Tie-1 has not been found, it has recently been demonstrated that Ang-1 can induce Tie-1 phosphorylation. ${ }^{13}$

The potential antiangiogenic role of the peptide somatostatin-14 (SRIF) and its analogues has received much attention, ${ }^{14}$ and it involves partial correction of systemic growth hormone dysregulation or inhibition of angiogenesis-associated factors. ${ }^{15-17}$ Of the five SRIF receptors mediating SRIF actions, $\mathrm{sst}_{2}$ is a likely candidate to mediate the angioinhibitory activity of SRIF. Indeed, analogues with high affinity for $\mathrm{sst}_{2}$, such as octreotide and BIM23027, counteract the growth factor-induced proliferation of bovine retinal endothelial cells under hypoxia. ${ }^{18}$ They are powerful inhibitors of neovascularization in models of proliferative retinopathies. ${ }^{15,19}$ In addition, octreotide inhibits the IGF-1-mediated induction of VEGF in human retinal pigment epithelial (RPE) cells. ${ }^{16}$ Moreover, octreotide retards retinopathy progression in diabetic patients in whom photocoagulation has failed. ${ }^{20}$ However, despite the growing use of $\mathrm{sst}_{2}$ agonists as antiangiogenic agents, the mechanisms by which these peptides inhibit the growth of new vessels has not been fully delineated.

Both $\mathrm{sst}_{1}$ - and $\mathrm{sst}_{2}$-knockout (KO) mice have been generated. ${ }^{21,22}$ In their retinas, we have found that sst $_{1}$ loss causes an increased expression and function of $\mathrm{sst}_{2} \cdot{ }^{23-28}$

In the present study, retinas of $\mathrm{sst}_{1}$ - and $\mathrm{sst}_{2}-\mathrm{KO}$ mice were rendered hypoxic and were used to investigate whether altered levels of $s t_{2}$ play a role in regulating retinal angiogenesis and its associated factors. Our hypothesis was that, compared with wild-type (WT) retinas, the lack of $\mathbf{s s t}_{2}$ is associated with heavier effects of hypoxia, whereas a chronic overexpression of $\mathrm{sst}_{2}$ (as in $\mathrm{sst}_{1}-\mathrm{KO}$ retinas) should attenuate these effects.

\section{Methods}

\begin{abstract}
Animals
Experiments were performed on 128 mice of WT (C57BL/6) and sst ${ }_{1}$ or $\mathrm{sst}_{2}$-KO strains of both sexes at postnatal day (PD)17 (6 g body weight). In some experiments designed to evaluate development of retinal vasculature, five PD12 mice for each strain were also used. sst $_{1}$ and $\mathrm{sst}_{2}-\mathrm{KO}$ mice were generated as previously reported. ${ }^{21,22}$ Experiments were performed in agreement with the ARVO Statement for the Use of Animals in Ophthalmic and Vision Research and in compliance with the Italian law on animal care 116/1992 and EEC/609/86. All efforts were made to reduce the number of animals used.
\end{abstract}

